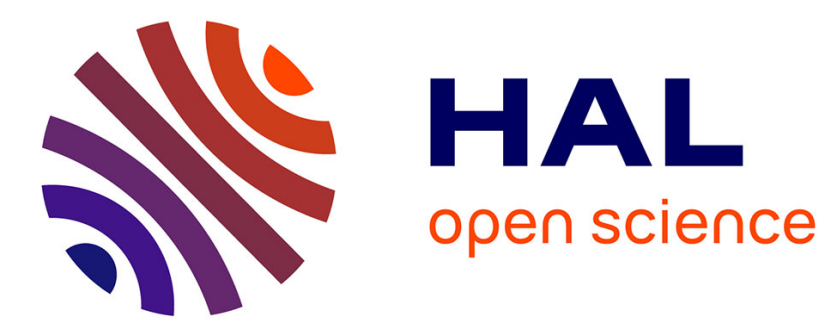

\title{
Magnetic Transitions and Magneto-Transport in Epitaxial Pr0.5Sr0.5MnO3 Thin Films
}

P. Wagner, V. Metlushko, M. van Bael, R. Vullers, L. Trappeniers, A. Vantomme, J. Vanacken, G. Kido, V. Moshchalkov, Y. Bruynseraede

\section{- To cite this version:}

P. Wagner, V. Metlushko, M. van Bael, R. Vullers, L. Trappeniers, et al.. Magnetic Transitions and Magneto-Transport in Epitaxial Pr0.5Sr0.5MnO3 Thin Films. Journal de Physique IV Proceedings, 1996, 06 (C3), pp.C3-309-C3-314. 10.1051/jp4:1996347 • jpa-00254265

\section{HAL Id: jpa-00254265 https://hal.science/jpa-00254265}

Submitted on 1 Jan 1996

HAL is a multi-disciplinary open access archive for the deposit and dissemination of scientific research documents, whether they are published or not. The documents may come from teaching and research institutions in France or abroad, or from public or private research centers.
L'archive ouverte pluridisciplinaire HAL, est destinée au dépôt et à la diffusion de documents scientifiques de niveau recherche, publiés ou non, émanant des établissements d'enseignement et de recherche français ou étrangers, des laboratoires publics ou privés. 


\title{
Magnetic Transitions and Magneto-Transport in Epitaxial Pro.5 $\mathrm{Sr}_{0.5} \mathrm{MnO}_{3}$ Thin Films
}

P. Wagner*, V. Metlushko*, M. Van Bael*, R.J.M. Vullers*, L. Trappeniers*, A. Vantomme**, J. Vanacken*,***, G. Kido***, V.V. Moshchalkov* and Y. Bruynseraede*

* Laboratorium voor Vaste-Stoffysica en Magnetisme, Katholieke Universiteit Leuven, Celestijnenlaan 200 D, 3001 Leuven, Belgium

** Instituut voor Kern-en Stralinsfysica, Katholieke Universiteit Leuven, Celestijnenlaan 200 D, 3001 Leuven, Belgium

*** Tsukuba Magnet Laboratories, National Research Institute for Metals, Sengen 1-2-1, Tsukuba Science City, Ibaraki 305, Japan

\begin{abstract}
Epitaxial thin films of the magnetic perovskite $\operatorname{Pr}_{0.5} \mathrm{Sr}_{0.5} \mathrm{MnO}_{3}$ were prepared by dcmagnetron sputtering, structurally characterized by $\mathrm{x}$-ray diffraction and their physical properties investigated by magnetization and electrical transport measurements. Ferromagnetic ordering appears in zero-field at $263 \mathrm{~K}$, followed by a second transition into an antiferromagnetic state at $160 \mathrm{~K}$. The zero-field resistivity has a semiconducting behaviour according to Mott's law and becomes quasi-metallic only within the ferromagnetic state, whose temperature range can be extended by applying an external magnetic field. The negative magnetoresistance effect increases systematically with decreasing temperature and reaches a value of $700 \%$ at $1.5 \mathrm{~K}$ in a field of $12 \mathrm{~T}$. For temperatures below $75 \mathrm{~K}$ we observed additionally a memory effect, showing up as a persistent, field induced lowering of sample resistivity.
\end{abstract}

\section{INTRODUCTION}

It is well known that $\mathrm{LaMnO}_{3}$ is an antiferromagnetic (AFM) insulator, becoming a metallic conductor with a ferromagnetic ( $\mathrm{FM})$ ordering after doping with divalent ions $\left(\mathrm{Ca}^{2+}, \mathrm{Sr}^{2+}, \mathrm{Ba}^{2+}\right)$ on the site of trivalent La [1]. The discovery of giant negative magnetoresistance (GMR) in $\mathrm{La}_{0.67} \mathrm{Ba}_{0.33} \mathrm{MnO}_{3}$ and the structurally related $\mathrm{Nd}_{0.5} \mathrm{~Pb}_{0.5} \mathrm{MnO}_{3}[2,3]$ increased considerably the interest in these compounds. Above the Curie-temperature $T_{C}$ the doped La- and Nd-manganites are semiconductors and undergo at $T_{C}$ a transition to a ferromagnetic metallic state. This state persists down to the lowest temperatures, while the maximum of the GMR effect shows up around $T_{C}$. The conductivity is supposed to emerge primarily from hopping of magnetic polarons above $T_{C}[2,3]$, and from the double-exchange mechanism below $T_{C}[4]$.

Another manganite compound, $\operatorname{Pr}_{0.5} \mathrm{Sr}_{0.5} \mathrm{MnO}_{3}$, behaves differently: neutron-diffraction studies of polycrystalline $\mathrm{Pr}_{0.5} \mathrm{Sr}_{0.5} \mathrm{MnO}_{3}$ revealed again ferromagnetism below $200 \mathrm{~K}$, followed however by a coexistence of FM and AFM phases between 80 and $160 \mathrm{~K}$, and a purely AFM phase below $80 \mathrm{~K}$ [5]. Magnetization and transport measurements on a high-quality single crystal confirmed the two transitions with a $T_{C}=270 \mathrm{~K}$ and a Néel temperature $T_{N}=140 \mathrm{~K}[6]$. The conductivity was found to be metallic in the FM, and semiconducting in the AFM phase. Based on the jump-like resistivity increase at the transition from the FM to the AFM state, and the doping state with 0.5 charge carriers per $\mathrm{Mn}$ ion, it was speculated that the carriers in the $\mathrm{Pr}_{0.5} \mathrm{Sr}_{0.5} \mathrm{MnO}_{3}$ undergo a charge ordering transition into an insulating, antiferromagnetic Wigner crystal [6]. The origin of this AFM behaviour of the Pr-manganite is related to the weakening of the ferromagnetic $\mathrm{Mn}^{3+}-\mathrm{Mn}^{4+}$ double exchange coupling by the contraction of the chemical unit cell.

\section{SAMPLE PREPARATION}

Starting from a polycrystalline, disk-shaped target with nominal composition $\mathrm{P}_{0.50} \mathrm{Sr}_{0.50} \mathrm{Mn}_{1.00} \mathrm{O}_{\approx 3}$, thin films were deposited onto $\mathrm{Sr}^{\mathrm{r}} \mathrm{TiO}_{3}\left(\begin{array}{lll}1 & 0 & 0\end{array}\right)$ substrates by dc-magnetron sputtering in on-axis geo- 
metry. The target was prepared by mixing stoichiometric amounts of $\mathrm{Pr}_{6} \mathrm{O}_{11}, \mathrm{SrCO}_{3}$ and $\mathrm{MnCO}_{3}$, calcinating $\left(12 \mathrm{~h}\right.$ at $\left.900^{\circ} \mathrm{C}\right)$ and firing in air $\left(24 \mathrm{~h}\right.$ at $\left.1250^{\circ} \mathrm{C}\right)$ with intermediate grinding. After pulverizing the reacted material, the powder was pressed into a $2 \mathrm{~mm}$ thick disk (38 $\mathrm{mm}$ diameter) with $40 \mathrm{MPa}$ uniaxial pressure. The target was sintered in air for $24 \mathrm{~h}$ at $1250^{\circ} \mathrm{C}$ and an additional $6 \mathrm{~h}$ at $1350^{\circ} \mathrm{C}$. I )uring the sputtering of the films (120 min for a $3000 \AA$ thick layer) the discharge current was $100 \mathrm{~mA}(-290 \mathrm{~V})$ in a $1.5 \mathrm{hPa}$. flowing $\mathrm{O}_{2}$ atmosphere, and with a target-substrate distance of $20 \mathrm{~mm}$. The $\mathrm{SrTiO}_{3}$ substrates were glued with silver paint to an $\mathrm{Al}_{2} \mathrm{O}_{3}$ block, which was resistively heated up to $\approx 900^{\circ} \mathrm{C}$ in order to achieve epitaxial film growth. 'The actual temperature at the substrate surface is estimated to be $200^{\circ} \mathrm{C}$ lower, while higher deposition temperatures resulted in polycrystalline films. Although the phase formation is complete after deposition, the films were kept an additional $30 \mathrm{~min}$ in $10 \mathrm{hPa} \mathrm{O}_{2}$ at $900^{\circ} \mathrm{C}$, and $30 \mathrm{~min}$ in $500 \mathrm{hPa} \mathrm{O}_{2}$ at $600^{\circ} \mathrm{C}$, in order to achieve homogeneous oxidization.

The $x$-ray diffraction spectra show that the films have no extra phases and are $\vec{c}$-axis oriented with FWHM values for the $\Theta$-scan of the $\left(\begin{array}{lll}0 & 0 & 2\end{array}\right)$ reflection between $0.19^{\circ}$ and $0.39^{\circ}$. This indicates an excellent structural order, comparable to the $0.15^{\circ}$ for the $\left(\begin{array}{lll}2 & 0 & 0\end{array}\right)$ reflection of the single crystalline substrates. The lattice constant is $3.818 \AA \pm 0.002 \AA$, in good agreement with the half c-axis length determined with nentron diffraction, i. e. $7.644 \AA[5]$. The difference by a factor of two arises from the existence of a superstructure, caused by deviations from the ideal cubic perovskite lattice. The half diagonal of the $a b$ plane of the superstructure is $3.842 \AA$ [5], in good agreement with the lattice constant of the $\mathrm{SrTiO}_{3}$ substrate $(3.905 \AA)$. Therefore a strong in-plane texture can be expected, and $\vec{a}$ - or $\vec{b}$-axis oriented film growth have not been observed. Scanning electron microscopy showed no trace of particles on the mirror-like film surfaces, while the average roughness of a $1 \mu^{2}{ }^{2}$ surface obtained with atomic force microscopy is $40 \AA$ for a $3000 \AA$ thick film. Composition analysis by means of Rutherford backscattering revealed the ratio $\operatorname{Pr}_{0.5} \operatorname{Sr}_{0.5} \mathrm{Mn}_{1.25} \mathrm{O}_{\approx 3}$ with an uncertainty of less than $10 \%$ for the cations. The correct concentration ratio of $\mathrm{Pr}^{3+}$ and $\mathrm{Sr}^{2+}$ is the essential precondition for the proposed charge ordering transition.

\section{MAGNETIC PROPERTIES}

The in-plane sample magnetization $M$ was measured with a Quantum Design SQUID at fields between $20 \mathrm{mT}$ and $5 \mathrm{~T}$. Prior to applying a field. the sample was cooled from $300 \mathrm{~K}$ to $5 \mathrm{~K}$ in zero field in order to ensure equivalent preconditions for each measurement. The measurements were then performed by warming up to $300 \mathrm{~K}$ with subsequent cooling to $5 \mathrm{~K}$, while Fig. 1 shows representative $M\left(T^{\prime}\right)$ curves for $20 \mathrm{~m}^{\prime} \mathrm{I}, 50 \mathrm{~m} \mathrm{I}^{\prime}$ and $1 \mathrm{~T}$. Higher magnetic fields did not substantially alter the appearance of the data for $1 \mathrm{~T}$.

The mean-field ferromagnetic Curie temperature $T_{C}$ was determined by linearly extrapolating the $M(T)^{-1}$ data to zero. For $20 \mathrm{mT}$, close to the zero-field limit, we obtained $T_{C}=263 \mathrm{~K}$, comparable to $270 \mathrm{~K}$ as reported for the $\operatorname{Pr}_{0.5} \mathrm{Sr}_{0.5} \mathrm{MnO}_{3}$ single crystal [6]. By applying external fields, $T_{C}$ is shifted slightly to higher temperatures. Although in the presence of fields above $20 \mathrm{mT}$ no clear Curie-Weiss behaviour is found in the data, this shift is also reflected by the shift of $T_{C}^{*}$, defined as the high temperature inflection point of $M(T)$, see Fig. 1. The transition to the AF state occurs around $T_{N}=160 \mathrm{~K}\left(B_{e x t}=20 \mathrm{~m}^{\top} \mathrm{T}\right)$ as clearly indicated by the reduction. of $M$ with decreasing $\mathrm{T}$. This implies that the $\mathrm{Mn}^{3+}$ and $\mathrm{Mn}^{4+}$ ions form independently two AF sublattices, if we assume different moments for $\mathrm{Mn}^{4+}\left(4.0 \mu_{B}\right)$ and $\mathrm{Mn}^{3+}\left(5.0 \mu_{B}\right)$, according to ref. [7]. With increasing external field the local magnetization maximum at $T_{N}$ is less pronounced and shifts to slightly lower temperatures. The onset of the AF behaviour (Fig. 1) is at all fields also accompanied by a splitting of the cooling and the warming magnetization data, reflecting the occurence of hysteresis effects. We note that the magnetic moment of the sample measured in $1 \mathrm{~T}$ at $5 \mathrm{~K}\left(1.5 \cdot 10^{-3} \mathrm{emu}\right)$ corresponds to a moment of only $0.62 \mu_{B}$ per $\mathrm{Mn}$ ion, which is below the above mentioned values. The occurence of relatively small $\mathrm{Mn}$ moments is however also known from La-manganites in form of thin films. At $T_{A} \approx 45 \mathrm{~K}$ 
(see Fig. 1) a small jump in the magnetization is observed, especially in the cooling runs. 'Two mechanisms may cause this jump: i) an ordering of the $\mathrm{Pr}^{3+}$ moments, and ii) the presence of magnetically ordering second phases. Concerning the first possibility, it is known that the Pr-moments in $\mathrm{PrBa}_{2} \mathrm{Cu}_{3} \mathrm{O}_{7}$ undergo antiferromagnetic ordering with a Néel temperature of $17 \mathrm{~K}[8]$, comparable to the $45 \mathrm{~K}$ observed for the $\mathrm{Pr}_{0.5} \mathrm{Sr}_{0.5} \mathrm{MnO}_{3}$ films. Also in the $\mathrm{Nd}_{0.5} \mathrm{~Pb}_{0.5} \mathrm{MnO}_{3}$ system a susceptibility anomaly showed up at $30 \mathrm{~K}$, which was associated with ordering in the $\mathrm{Nd}^{3+}$ lattice $\left(3.5 \mu_{B}\right)[3]$. A possible second phase could consist of amorphous traces of the Mn-rich $\operatorname{PrMn}_{2} \mathrm{O}_{5}$ with $T_{N}=46 \mathrm{~K}$ [9]. XRD reflections corresponding to the lattice constants of this compound were not detected for the sample under investigation, therefore we conclude the volume fraction to be probably below $10 \%$.

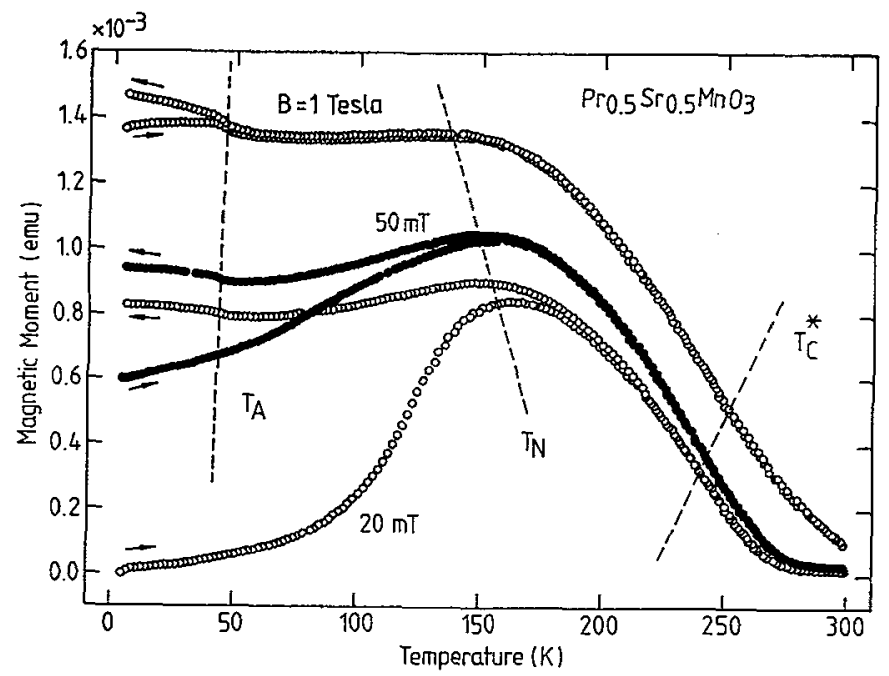

Figure 1: In-plane magnetic moment of a $\mathrm{Pr}_{0.5} \mathrm{Sr}_{0.5} \mathrm{MnO}_{3}$ film vs. temperature for fields of $20 \mathrm{mT}, 50 \mathrm{mT}$, and $1 \mathrm{~T}$ in warming and cooling runs as indicated by arrows. The lines suggest the positions of (i) the Curie temperature $T_{C}^{\star}$ of ferromagnetism, (ii) the onset temperature $T_{N}$ of antiferromagnetic correlations, and (iii) the low temperature anomaly $T_{A}$ as discussed in the text.

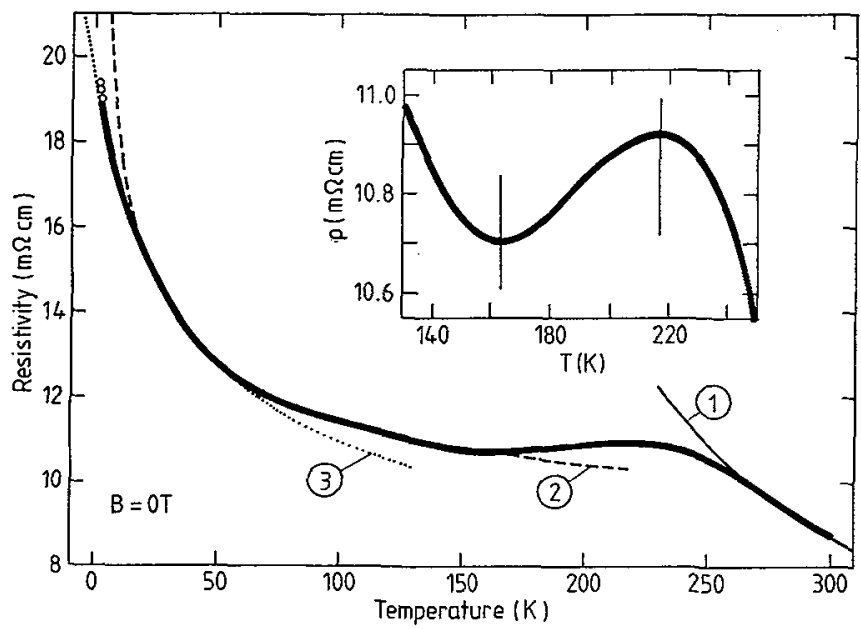

Figure 2: Zero-field resistivity as a function of temperature of a $\operatorname{Pr}_{0.5} \mathrm{Sr}_{0.5} \mathrm{MnO}_{3}$ film. The fit functions are based on (1) thermally activated nearest neighbour hopping in the paramagnetic phase, (2) variable range hopping in the antiferromagnetic phase below $160 \mathrm{~K}$, and $(3)$ the empirical low temperature scaling $\rho \propto \exp \left(\Theta_{1} /\left(T+\Theta_{2}\right)\right)$. The insert shows a detail of the metallic (ferromagnetic) regime. 


\section{CONDUCTIVITY MECHANISM IN ZERO FIELD}

The resistivity measurements were performed on a stripe cut from a $\operatorname{Pr}_{0.5} \mathrm{Sr}_{0.5} \mathrm{MnO}_{3}$ film $(10 \mathrm{~mm}$ long, $2.8 \mathrm{~mm}$ wide) after evaporating four gold contacts in one line across the sample and annealing them in air for $15 \mathrm{~min}$ at $700^{\circ} \mathrm{C}$. These contacts had a resistance below $1 \Omega$ and the measurements were carried out with an ac-bridge $(28 \mathrm{~Hz})$ at an effective excitation current of $0.3 \mu \mathrm{A}$. The current direction was within the $a b$ plane of the film, parallel with the field axis of the cryostate, providing that the measurements of Sect. 5 were performed in the Lorentz-force free configuration.

Figure 2 shows the temperature dependence of the zero-field resistivity, with a magnification of the metallic-like region between $160 \mathrm{~K}$ and $220 \mathrm{~K}$ in the insert. This temperature dependence corresponds more or less to the single crystal data reported by Tomioka et al. [6], including comparable resistivity values, except for the smooth quasi-metallic to semiconducting transition at $160 \mathrm{~K}$. Above the metallic-like region $(\mathrm{T}>250 \mathrm{~K}$ ) the resistivity scales with thermally activated nearest neighbour hopping $\left(\rho \propto \exp \left(U / k_{B} T\right), U \approx 30 \mathrm{meV}\right)$, which can be attributed to magnetic polarons, as assumed for the doped $\mathrm{Nd}$ - and La-manganites $[2,3]$. Below the metallic region $(50 \mathrm{~K}<\mathrm{T}<160 \mathrm{~K})$ a semiconducting behaviour is observed in accordance with Mott's law for variable range hopping (VRH), i. e. $\log \rho \propto(\Theta / T)^{1 / 4}[10]$. Below $50 \mathrm{~K}$ the resistivity starts to deviate slightly from Mott's law and scales with $\log \rho \propto \Theta_{1} /\left(T+\Theta_{2}\right)\left(\Theta_{1}=54.9 \mathrm{~K}, \Theta_{2}=60.0 \mathrm{~K}\right)$. This temperature dependence resembles the susceptibility of an antiferromagnet above $T_{N}$ and remains valid also in the presence of external magnetic fields, with slightly other values for $\Theta_{1}$ and $\Theta_{2}$. Although there is no theoretical model for this $\rho(T)$ behaviour at $T<50 \mathrm{~K}$, the correspondence of the empirical relation with the data is excellent. The existence of two different scaling regimes below $160 \mathrm{~K}$ indicates again a change in magnetic ordering around $50 \mathrm{~K}$, in accordance with the magnetization data.

\section{GIANT NEGATIVE MAGNETORESISTIVITY}

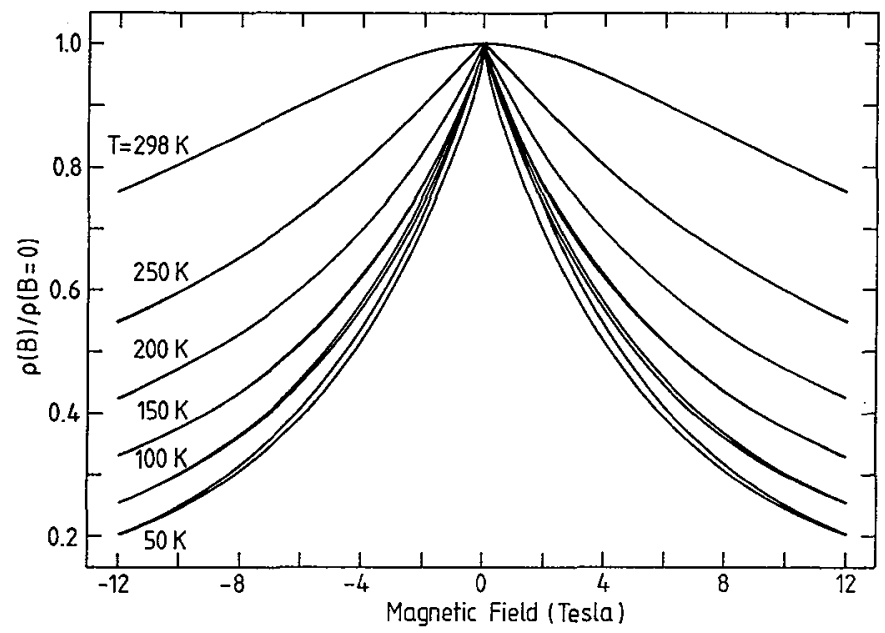

Figure 3: Field dependence of the magnetoresistivity at different temperatures. The curvature is positive for $298 \mathrm{~K}$ and negative for all temperatures below, indicating a unique GMR mechanism for $T \leq 250 \mathrm{~K}$. Hysteretic behaviour is visible up to $150 \mathrm{~K}$, with coercitive fields below $100 \mathrm{mT}$.

Resisistivity measurements at fixed temperatures are shown in Fig. 3. Each curve consists of four field sweeps in the sense $0 T \rightarrow 12 T \rightarrow 0 T \rightarrow-12 T \rightarrow 0 T$, starting with a zero-field cooled sample. The curves for $50 \mathrm{~K}, 100 \mathrm{~K}$ and $150 \mathrm{~K}$ exhibit a splitting due to hysteresis which becomes even more pronounced at $5 \mathrm{~K}$. The data at $5 \mathrm{~K}$ are omitted, since the memory effect at low temperatures 
(see Sect. 6) leads to further complications in interpreting the $\rho(B)$ changes. From the difference in curvature of the $\rho(B)$ data at $298 \mathrm{~K}$ and the lower temperature data we may conclude that a different mechanism is present in the paramagnetic and the ordered states. Double logarithmic plots of the field induced conductivity vs. field revealed the scaling:

$$
\sigma(B, T)=\sigma(B=0, T)+\sigma^{\star}\left(T^{\prime}\right) \cdot B^{\alpha}
$$

At $298 \mathrm{~K}$ the exponent $\alpha \approx 1.82$, a value close to 2 , which was reported for the scattering of charge carriers by independent spins [11,12]. For all other temperatures up to $250 \mathrm{~K}, \alpha$ varies between 0.99 and 1.20, while deviations are present mainly in the antiferromagnetic phase. Especially at $200 \mathrm{~K}$, still in the ferromagnetic state the conductivity increase remains strictly linear for fields as high as $25 \mathrm{~T}$ [13]. It should also be pointed out that the scaling with Eq. 1 can only be a low field approximation, in the sense that saturation tendencies of the GMR effect, especially visible at the low temperature curves of Fig. 3, are not taken into account.

\section{MEMORY EFFECTS}

At temperatures below $T_{M}=75 \mathrm{~K}$ we observe an irreversible change of the sample resistivity after application of high external fields. The resistivity at e. g. $5 \mathrm{~K}$, measured directly after zero-field cooling. is $17 \%$ higher than the highest resistivities observed at the coercitive fields in a resistive hysteresis loop after a field sweep to $12 \mathrm{~T}$. This result confirms similar observations of memory effects for $\mathrm{Nd}_{0.7} \mathrm{Sr}_{0.3} \mathrm{MnO}_{3}$ and $\mathrm{La}_{0.67} \mathrm{Ca}_{0.33} \mathrm{MnO}_{3}$ thin films [12,14], as well as $\mathrm{Pr}_{0.7} \mathrm{Sr}_{0.04} \mathrm{Ca}_{0.26} \mathrm{MnO}_{3}$ bulk samples [15]. In these compounds, with comparatively high resistivities after $Z F$ cooling, the freld induced resistivity decay reaches several orders of magnitude. The most probable origin for this memory effect seems to be a persistent alteration of the spin structure, as found by neutron diffraction on $\mathrm{Pr}_{0.7} \mathrm{Ca}_{0.3} \mathrm{MnO}_{3}$ single crystals [16]. The antiferromagnetic spin arrangement, which is also the low-temperature spin structure of our $\mathrm{Pr}_{0.5} \mathrm{Sr}_{0.5} \mathrm{MnO}_{3}$ samples, was modified into a ferromagnetic structure, encouraging carrier transport by means of the double exchange mechanism. The temperature dependence of the memory effect was studied by first zero-field cooling of the sample to $4.2 \mathrm{~K}$ and applying an external field of $30 \mathrm{~T}$. After sweeping the external field to zero, the resistivity was measured by warming $\left(\rho_{W}\right)$ to room temperature and cooling $\left(\rho_{C}\right)$ back to $4.2 \mathrm{~K}$, where the memory effect $\left(\rho_{C}-\rho_{W}\right) / \rho_{C}$ reaches over $30 \%$ (see Fig. 4).

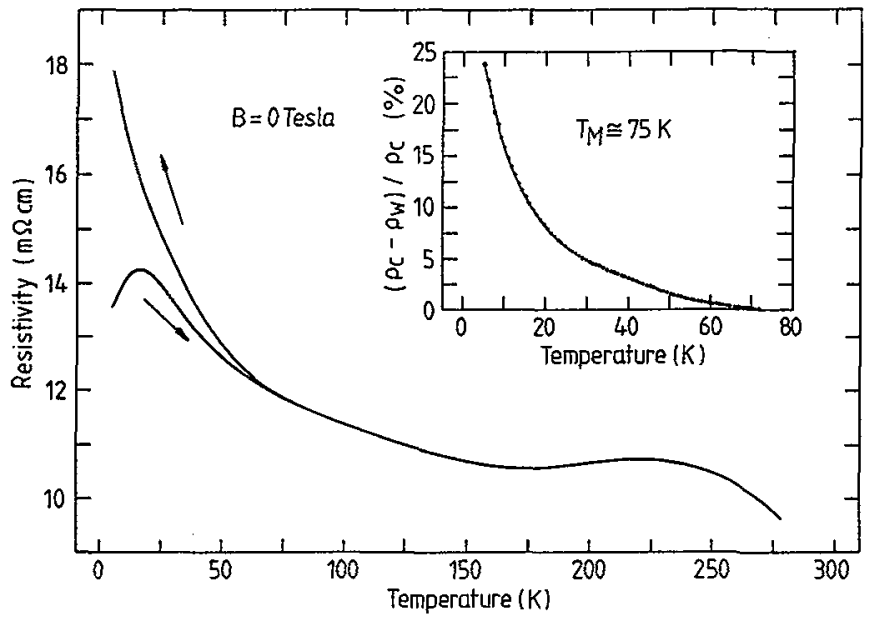

Figure 4: Temperature dependence of resistivity in the remanent cryostat field after exposing the sample at $4.2 \mathrm{~K}$ to an external field of $30 \mathrm{~T}$. 'The direction of warming and cooling is indicated by arrows, while the difference between both curves vanishes at $75 \mathrm{~K}$. 
As can be seen from the insert of Fig. 4 , the difference between the two measurements vanishes above $70 \mathrm{~K}$. We should note here, that the temperature of this irreversibility point does neither agree with the upper temperature limit for the low-temperature resistivity scaling from Sect. 4, nor with a characteristic anomaly of the magnetization curves in Sect. 3. However, it agrees well with the complete vanishing of a ferromagnetic signal in the neutron diffraction spectra of $\mathrm{Pr}_{0.5} \mathrm{Sr}_{0.5} \mathrm{MnO}_{3}$ powder at $80 \mathrm{~K}[5]$.

\section{Conclusions}

Thin films of $\mathrm{Pr}_{0.5} \mathrm{Sr}_{0.5} \mathrm{MnO}_{3}$ were prepared in situ with strong $\vec{c}$-axis orientation, and are characterized with respect to morphology and surface properties. The zero-field resistivity showed semiconducting behaviour according to nearest neighbour hopping in the paramagnetic, and variable range hopping in the antiferromagnetic state. Metallic conductivity was only observed in the temperature regime between $160 \mathrm{~K}$ and $220 \mathrm{~K}$, where magnetization measurements reveal a ferromagnetic state. Surprisingly, the CMR mechanism in the antiferromagnetic state seems to be of a similar origin as for the ferromagnet, since both obey in good approximation the same scaling law for the conductivity increase, which is almost directly proportional to the strength of the externally applied field. We want to point out that for potential field sensing purposes it seems to be more interesting to use antiferromagnetic compounds since they exhibit GMR not only around the ferromagnetic transition temperature as the doped La-manganites, but at all temperatures below room temperature.

\section{Acknowledgements}

This work is supported by the Belgian Incentive High- $T_{c}$ Superconductivity Programme (SU.01), the National Fund for Scientific Research (NFWO) and the Inter-University Attraction Poles (IUAP). P. W. and V. M. are postdoctoral fellows of the NFWO and the Research Council of KU Leuven.

\section{References}

[1] Jonker G. H. and Van Santen J. H., Physica 16. 337 and 599 (1950).

[2] von Helmolt R. et al., Phys. Rev. Lett. 71, 2331 (1993).

[3] Kusters R. M. et al., Physica B 155, 362 (1989).

11] Zener. C., Phys. Rev. 82, 403 (1951), de Gennes P. G., Phys. Rev. 118. 141 (1960).

[5] Knižek K. et al., Journ. of Sol. Stat. Chem. 100. 292 (1992).

[6] 'Tomioka Y. et al., Phys. Rev. Lett. 74, 5108 (1995).

[7] Ashcroft N. W. and Mermin N. D.. Solid State Physics, Saunders College, Philadelphia (1976).

[8] Kebede A. et al.. Phys. Rev. B 40, 4453 and l. Felner et al., Phys. Rev. B 40, 6739 (1989).

[9] Bertaut F. F. et al.. Bull. Soc. Chim. France, 1132 (1965).

[10] Mott N. F. and Davis E. A. Electronic Processes in Non-Crystalline Materials, Oxford (1971).

[11] Sun J. Z. et al., Appl. Phys. Lett. 67, 2726 (1995).

[12] Xiong G. C. et al., Appl. Phys. Lett 67, 3031 (1995).

[13] Vanacken d. et al., to be published (1996).

[14] von Helmolt R. et al., Appl. Phys. Lett. 67, 2093 (1995).

[15] Maignan A. et al., Sol. Stat. Comm. 96, 623 (1995).

[16] Yoshizawa H. et al., Phys. Rev. B 52, 13145 (1995). 